\title{
Fast Cardinal Interpolation
}

\author{
Eric M. Guild, Eric C. Like and Steven C. Gustafson*
}

Air Force Institute of Technology, AFIT/ENG, Wright-Patterson AFB, OH 45433, USA

\begin{abstract}
A computationally fast and optimally smooth method for generating a probability density of $\mathrm{y}$ given $\mathrm{x}$ that models given data points is described and illustrated. This method interpolates in that the mean function intersects the points and the variance function is zero at the points. It is fast and optimal in that it is produced by the smallest number of maximally-smooth Gaussian radial interpolators for which the extrapolated density has the mean and variance of the classic linear model.
\end{abstract}

\section{INTRODUCTION}

Cardinal interpolation refers to a class of recentlyintroduced methods for generating probability densities of $y$ given $\mathrm{x}$ that model given $(\mathrm{x}, \mathrm{y})$ data points [1-3]. A cardinal density interpolates in that it has a mean function which intersects the points and a variance function which is zero at the points. A cardinal density also extrapolates to the density of the classic linear model for which the mean function is the least squares line of the points. Cardinal densities have various optimality properties depending on their type, but typically they are non-Gaussian and thus may be regarded as generalizations of well-known Gaussian process densities [4].

The generation of cardinal densities can be computationally intensive. This article describes and illustrates an interpolating cardinal density that is fast (not computationally intensive) and that has desirable optimality properties.

\section{MOTIVATION}

Interpolating cardinal densities investigated to date [1-3] have all been generated using a weighted sum of a large number of basis functions. Each basis function has the form (although other forms could be used) of a line plus a sum of same-variance Gaussians, and there is one Gaussian for each data point with its mean at the point. The intercept and slope of the line differs for different basis functions, but for each basis function the Gaussian amplitudes are such that the points are interpolated (intersected) and the variance is such that the roughness of the basis function is minimized, where roughness has the classic integrated squared second derivative definition (although other definitions could be used).

Weights are assigned to the basis functions such that the cardinal density extrapolates to the density of the classic linear model for which the mean function is the least squares

*Address correspondence to this author at the Air Force Institute of Technology, AFIT/ENG, Wright-Patterson AFB, OH 45433, USA;

E-mails: Steven.Gustafson@afit.edu, gustafson.steven@gmail.com line of the points and the variance function is quadratic. Since the basis functions are lines upon extrapolation (i.e., for large magnitude $\mathrm{x}$ ), a straightforward Bayesian analysis yields the weights for a large number of lines with intercepts and slopes uniformly spaced over large domains.

In this analysis the classic linear model density $\mathrm{p}(\mathrm{y} \mid \mathrm{x}, \mathrm{D})$ of $y$ given $x$ and the points $D$ is $p(y \mid x, D)=\int p(y \mid x, w)$ $p(w \mid D) d w$, where $w$ is a vector of weights. Here $p(y \mid x, w)$ is chosen to be a Gaussian proportional to $\exp [-(\mathrm{y}-\mathrm{a}-$ $\left.\mathrm{bx})^{2} /\left(2 \sigma^{2}\right)\right]$, and by Baye's rule $\mathrm{p}(\mathrm{w} \mid \mathrm{D})$ is proportional to $\mathrm{p}(\mathrm{D} \mid \mathrm{w}) \mathrm{p}(\mathrm{w})$, where prior density $\mathrm{p}(\mathrm{w})$ is chosen to be uninformative and thus constant, and as a consequence of the Gaussian form for $\mathrm{p}(\mathrm{y} \mid \mathrm{x}, \mathrm{w}), \mathrm{p}(\mathrm{w} \mid \mathrm{D})$ is proportional to $\exp [-$ $\left.\Sigma\left(y_{i}-\mathrm{a}-\mathrm{bx}_{\mathrm{i}}\right)^{2} /\left(2 \sigma^{2}\right)\right]$. These stipulations enable analytic evaluation of the above integral, with the result that the classic linear model density is Gaussian with a mean that is the least squares line of the points and a variance that is the quadratic function $\sigma^{2}+\sigma^{2} / \mathrm{n}+\left(\sigma^{2} / \sigma_{\mathrm{x}}{ }^{2}\right) \mathrm{x}^{2}$, where $\sigma_{\mathrm{x}}{ }^{2}$ is the variance of the point $x$ values.

With the above choices for the classic linear model, the above integral may be expressed in a "function space" form [4] proportional to $\iiint \delta(\mathrm{y}-\mathrm{a}-\mathrm{bx}-\mathrm{c}) \cdot \exp \left\{-\left[\Sigma\left(\mathrm{y}_{\mathrm{i}}-\mathrm{a}-\mathrm{bx}_{\mathrm{i}}\right)^{2}\right.\right.$ $\left.\left.+\mathrm{c}^{2}\right] /\left(2 \sigma^{2}\right)\right\} \mathrm{da} \mathrm{db} \mathrm{dc}$, where the integrals are over infinite domains. This form shows that the classic linear model density consists of the average of an infinite number of "delta density lines" (i.e., a line for all possible choices of a, $\mathrm{b}$, and $\mathrm{c})$, where each line has the weight $\exp \left\{-\left[\Sigma\left(\mathrm{y}_{\mathrm{i}}-\mathrm{a}-\right.\right.\right.$ $\left.\left.\left.\mathrm{bx}_{\mathrm{i}}\right)^{2}+\mathrm{c}^{2}\right] /\left(2 \sigma^{2}\right)\right\}$.

In previous work [1-3], an interpolating cardinal density was obtained as a weighted average of a large number of basis functions, each formed by adding Gaussians to a delta density line (e.g., 27,000 lines formed from 30 uniformlyspaced values of $a, b$, and $c$ ). This paper describes and illustrates a method that generates an interpolating cardinal density from the minimum possible number of lines, which (as shown below) is four, where each line has the same weight. The resulting fast cardinal interpolation density is not the same as the density obtained using a large number of basis functions. However, it is readily computed and has the all required properties, including the property that its four 
point-centered same-variance Gaussian basis functions have minimal roughness.

\section{DESCRIPTION}

A fast and optimal interpolating cardinal density may be generated as follows:

1. Normalize the given points to have zero mean and unit variance in both $\mathrm{x}$ and $\mathrm{y}$ and to have the $\mathrm{x}$ axis as their least squares line. Thus, without loss of generality, the coordinates of the original points are linearly translated, rotated, and scaled so that the resulting points are $\left(\mathrm{x}_{\mathrm{i}}, \mathrm{y}_{\mathrm{i}}\right), \mathrm{i}=1,2, \ldots, \mathrm{n}$, with $\Sigma \mathrm{x}_{\mathrm{i}}=$ $\Sigma \mathrm{y}_{\mathrm{i}}=0, \Sigma \mathrm{x}_{\mathrm{i}}^{2}=\Sigma \mathrm{y}_{\mathrm{i}}^{2}=\mathrm{n}$, and $\sum \mathrm{x}_{\mathrm{i}} \mathrm{y}_{\mathrm{i}}=0$.

2. Find the minimal set of basis lines. The interpolating cardinal density extrapolates to the density for the classic linear model. For the normalized points this density has zero mean for all $\mathrm{x}$ and has the quadratic variance $1+1 / n+x^{2} / n$ [4]. If this mean and variance are those of a set basis lines $y=a_{j}+b_{j} x, j=1,2, \ldots$, $\mathrm{m}$, then a mean of zero for all $\mathrm{x}$ requires $\sum \mathrm{a}_{\mathrm{j}}=\sum \mathrm{b}_{\mathrm{j}}=$ 0 , and a variance of $1+1 / \mathrm{n}+\mathrm{x}^{2} / \mathrm{n}$ requires $(1 / \mathrm{m}) \sum \mathrm{a}_{\mathrm{j}}^{2}$ $=1+1 / \mathrm{n},(1 / \mathrm{m}) \sum \mathrm{b}_{\mathrm{j}}{ }^{2}=1 / \mathrm{n}$, and $\sum \mathrm{a}_{\mathrm{j}} \mathrm{b}_{\mathrm{j}}=0$. These equations require at least four basis lines, and the minimal set has the four basis lines given by $\left(a_{j}, b_{j}\right)=$ $(\mathrm{a}, \mathrm{b}),(-\mathrm{a}, \mathrm{b}),(\mathrm{a},-\mathrm{b}),(-\mathrm{a},-\mathrm{b})$, where $\mathrm{a}=(1+1 / \mathrm{n})^{1 / 2}$ and $b=(1 / n)^{1 / 2}$.

3. Replace each of the four basis lines in the minimal set with the point-centered same-variance Gaussian radial interpolator that has least roughness. Thus $y=a$ $+\mathrm{bx}$ is replaced with $\mathrm{f}(\mathrm{x})=\mathrm{a}+\mathrm{bx}+\Sigma \mathrm{A}_{\mathrm{i}} \exp [-(\mathrm{x}-$ $\left.\left.\mathrm{x}_{\mathrm{i}}\right)^{2} /\left(2 \mathrm{~s}^{2}\right)\right]$, and similarly for the other three basis lines. Here the $A_{i}$ are such that the points are interpolated and are therefore found, for a given $s$, by solving $n$ linear equations in $n$ unknowns, i.e., the $A_{i}$ are such that $y_{i}=f\left(x_{i}\right)$. Also, $s$ is such that the integrated squared second derivative of $\mathrm{f}(\mathrm{x})$, which is a classic measure of roughness, is minimized, i.e., $\mathrm{s}$ is chosen to minimize $\int_{-\infty}^{\infty} \mathrm{f}(\mathrm{x})^{\prime \prime 2} \mathrm{dx}$. Thus there are $\mathrm{m}=4$ radial interpolators $\mathrm{f}_{\mathrm{j}}(\mathrm{x})$, each of which has distinct $\mathrm{A}_{\mathrm{i}}$ and $\mathrm{S}$.

4. Find the mean, variance, and skewness of the interpolating cardinal density at any $\mathrm{x}$ as $\mu=$ $(1 / \mathrm{m}) \Sigma \mathrm{f}_{\mathrm{j}}(\mathrm{x}), \quad \sigma^{2}=(1 / \mathrm{m}) \Sigma\left[\mathrm{f}_{\mathrm{j}}(\mathrm{x})-\mu\right]^{2}$, and $\gamma=$ $\left\{(1 / m) \Sigma\left[\mathrm{f}_{\mathrm{j}}(\mathrm{x})-\mu\right]^{3} / \sigma^{3}\right.$, where the minimal set $\mathrm{m}=4$ is used. Also, find the fast cardinal density of $y$ given $x$ as the standard skew-normal density $\mathrm{p}(\mathrm{y} \mid \mathrm{x})=(2 \pi)^{-1 / 2}$ $\omega^{-1} \exp \left[-(\mathrm{x}-\xi)^{2} /\left(2 \omega^{2}\right)\right] \cdot\left\{1+\operatorname{erf}\left[2^{-1 / 2} \alpha(\mathrm{x}-\xi) / \omega\right]\right\}$, where $|\delta|=(\pi / 2)^{1 / 2}|\gamma|^{1 / 3}\left[|\gamma|^{2 / 3}+(2-\pi / 2)^{2 / 3}\right]^{-1 / 2}$, $\operatorname{sign} \delta=\operatorname{sign} \gamma, \alpha=\delta\left(1-\delta^{2}\right)^{-1 / 2}, \omega=\sigma\left(1-2 \delta^{2} / \pi\right)^{-1 / 2}$, and $\xi=\mu-\omega \delta(2 / \pi)^{1 / 2}$. Note that the skew-normal density is a classic form that includes the Gaussian density as a special case but that allows asymmetry in the form of a smooth density "tail".

\section{PROPERTIES}

The fast cardinal density described above has the required properties: its mean function intersects the points, its variance function is zero at the points, and it extrapolates to the classic linear model density. Also, it is optimal in that it is generated using the smallest possible number of minimally rough Gaussian radial interpolators. Finally, it is computationally fast in that it requires only the solution of four sets of $n$ linear equations in $n$ unknowns (to determine the $A_{i}$ for each radial interpolator) plus, using analytic evaluation of the roughness integral, the solution of four nonlinear equations, each in one variable (to determine $\mathrm{s}$ for each radial interpolator).

Previous versions of cardinal interpolation [1-3] could be computationally intensive because they used large numbers of basis lines with wide ranges of intercepts and slopes. In a Bayesian analysis this choice approximates uniform and thus uninformative prior probability densities for the intercept and slope parameters. For fast cardinal interpolation the prior density of these parameters is parsimoniously chosen so that only the smallest possible set, i. e., $(a, b),(-a, b),(a,-b)$, and $(-\mathrm{a},-\mathrm{b})$, that yields the required properties is employed. This choice differs from the uniform prior that employs an indefinitely large set of basis functions, and thus the fast cardinal interpolation density does not necessarily approximate cardinal interpolation densities formed by averaging a large number of basis functions.

\section{ILLUSTRATION}

Fig. (1) shows three data points and their four radial interpolators. As described above, the points are normalized to have zero mean and unit variance in $\mathrm{x}$ and $\mathrm{y}$ and to have the $\mathrm{x}$ axis as their least squares line. Each radial interpolator is the sum of three Gaussians that extrapolate to one of the four basis lines, that have their means at the points, and that have the same variance, where this variance is such that each radial interpolator is maximally smooth, i.e., has least roughness. The four basis lines are such that their mean and variance are those of the classic linear model.

Fig. (2) shows the mean function and the mean function plus and minus the standard deviation function for the classic linear model and for fast cardinal interpolation.

Fig. (3) shows the fast cardinal interpolation density and the classic linear density at four values of $x$ : $1 / 3,2 / 3,4 / 3$, and $8 / 3$ of the distance to the positive-point $x$ value. Note that the fast cardinal interpolation density is skewed toward negative $y$ up to the positive-point $x$, that is otherwise skewed toward positive $y$, and that the skew vanishes for large $\mathrm{x}$.

Fig. (4) shows a communications signal consisting of thirty sample points that are normalized to have zero mean and unit variance in $\mathrm{x}$ and $\mathrm{y}$ and to have the $\mathrm{x}$ axis as their least squares line. As in Fig. (1), the points are interpolated with four (the smallest possible number) of least-rough Gaussian radial basis functions such that their mean and variance extrapolate to those of the classic linear model. The middle and bottom graphs show the standard deviation and the skewness of the basis functions. 


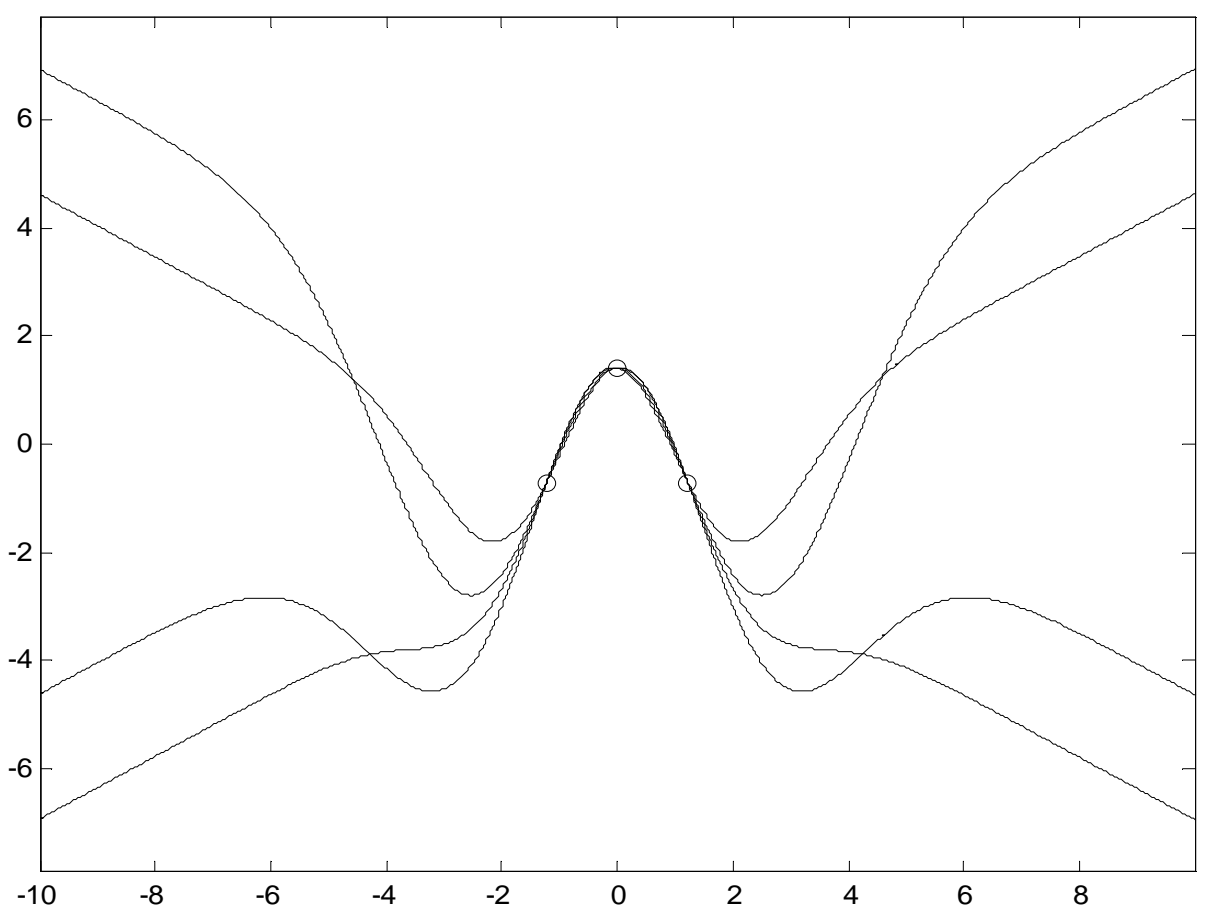

Fig. (1). Three data points and four radial interpolators. The points are normalized to have zero mean and unit variance in $\mathrm{x}$ and $\mathrm{y}$ and to have the $\mathrm{x}$ axis as their least squares line. Each radial interpolator is the sum of three Gaussians that all extrapolate to one of four basis lines, that have their means at the points, and that have the same variance, where this variance is such that the radial interpolator has least roughness. The four basis lines are such that their mean and variance are those of the classic linear model.

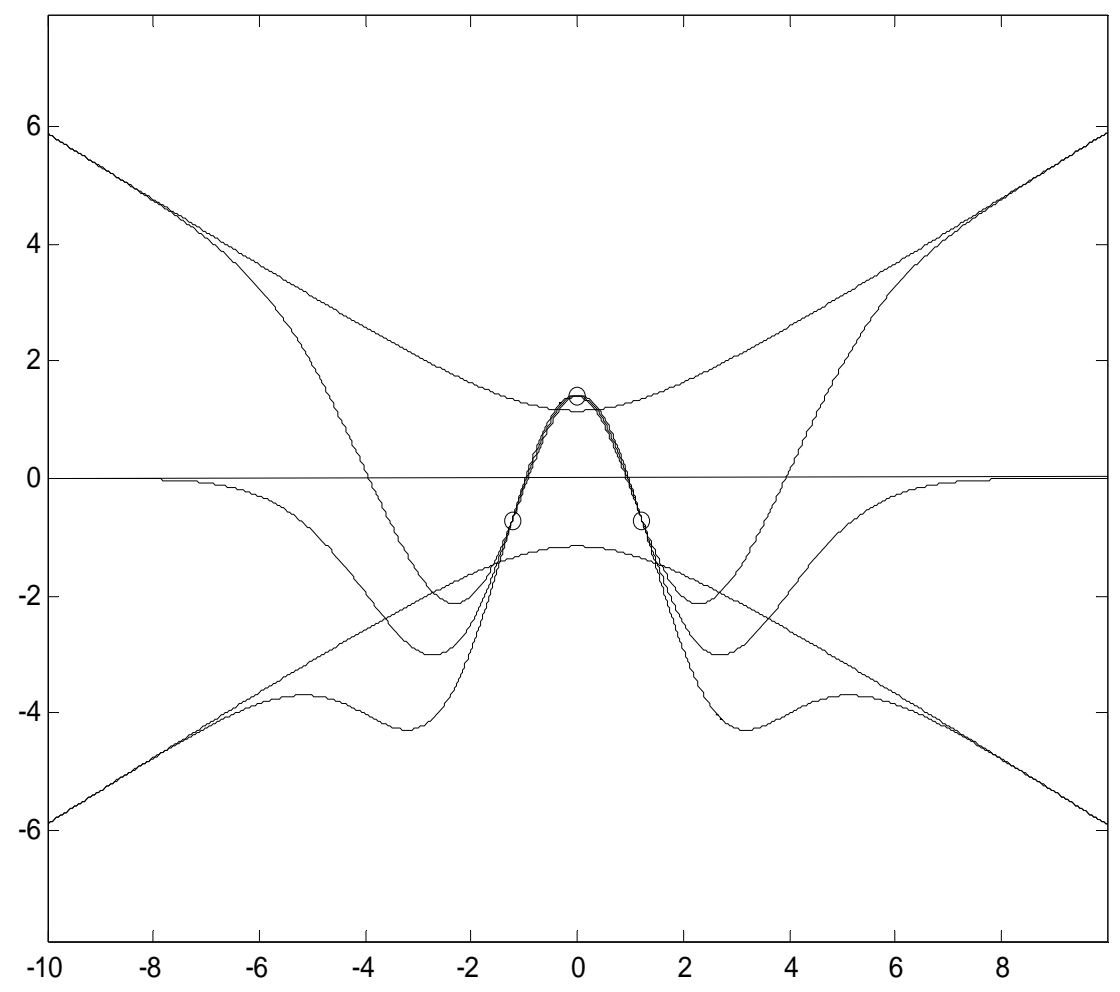

Fig. (2). The mean function and this function plus and minus the standard deviation function for the classic linear model and for fast cardinal interpolation. For the classic linear model the mean function is the $\mathrm{x}$ axis and the standard deviation is the square root of a quadratic function. For fast cardinal interpolation the mean function and this function plus and minus the standard deviation function all intersect the points. 

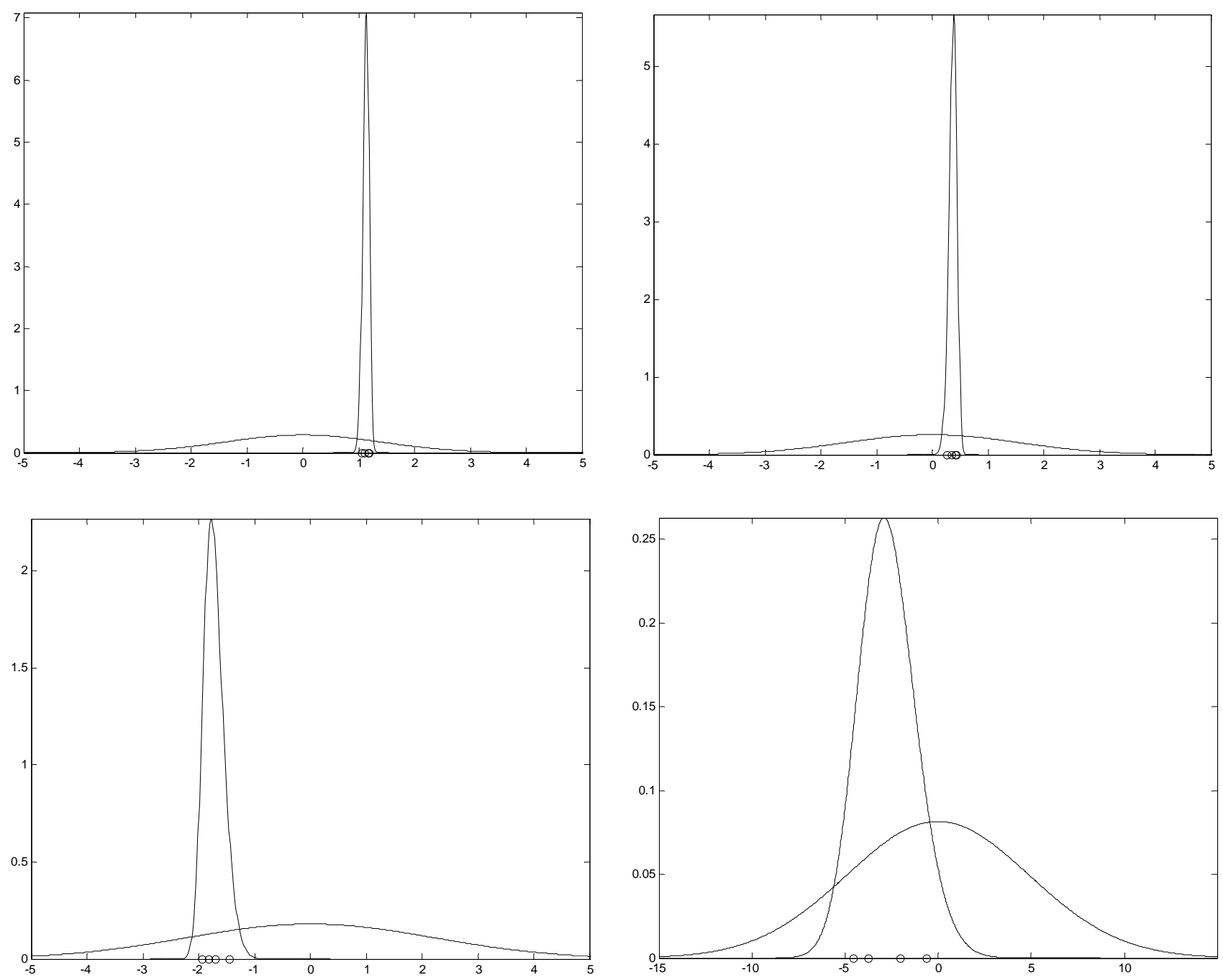

Fig. (3). The fast cardinal interpolation density and the classic linear model density at four values of $x$, and the $y$ values of the four radial interpolators at these $x$ values. The first two $x$ values are $1 / 3$ and $2 / 3$ of the positive-point $x$ value and yield the top left and right plots; the other two $x$ values are $4 / 3$ and $8 / 3$ of the positive-point $x$ value and yield the bottom left and right plots. The fast cardinal interpolation density is skewed toward negative y in the top left and right plots (skewness of -.268 and -.430, respectively) and is skewed toward positive y in the bottom left and right plots (skewness of .469 and .211 , respectively). The classic linear model density is Gaussian with zero mean in all plots.

\section{CONCLUSION}

Unlike previously-reported versions of cardinal interpolation, the method reported here is computationally fast in that it is generated using the smallest number (four) of radial interpolators needed to yield the required properties. These properties are that the mean function intersects the points, the variance function is zero at the points, and extrapolation is to the classic linear model density.
Furthermore, fast cardinal interpolation is optimal in that each of the four point-centered same-variance Gaussian radial interpolators has least roughness.

\section{ACKNOWLEDGEMENT}

The views expressed in this paper are those of the authors and do not reflect the official policies or position of the United States Air Force, the US Department of Defense, or the US Government. 

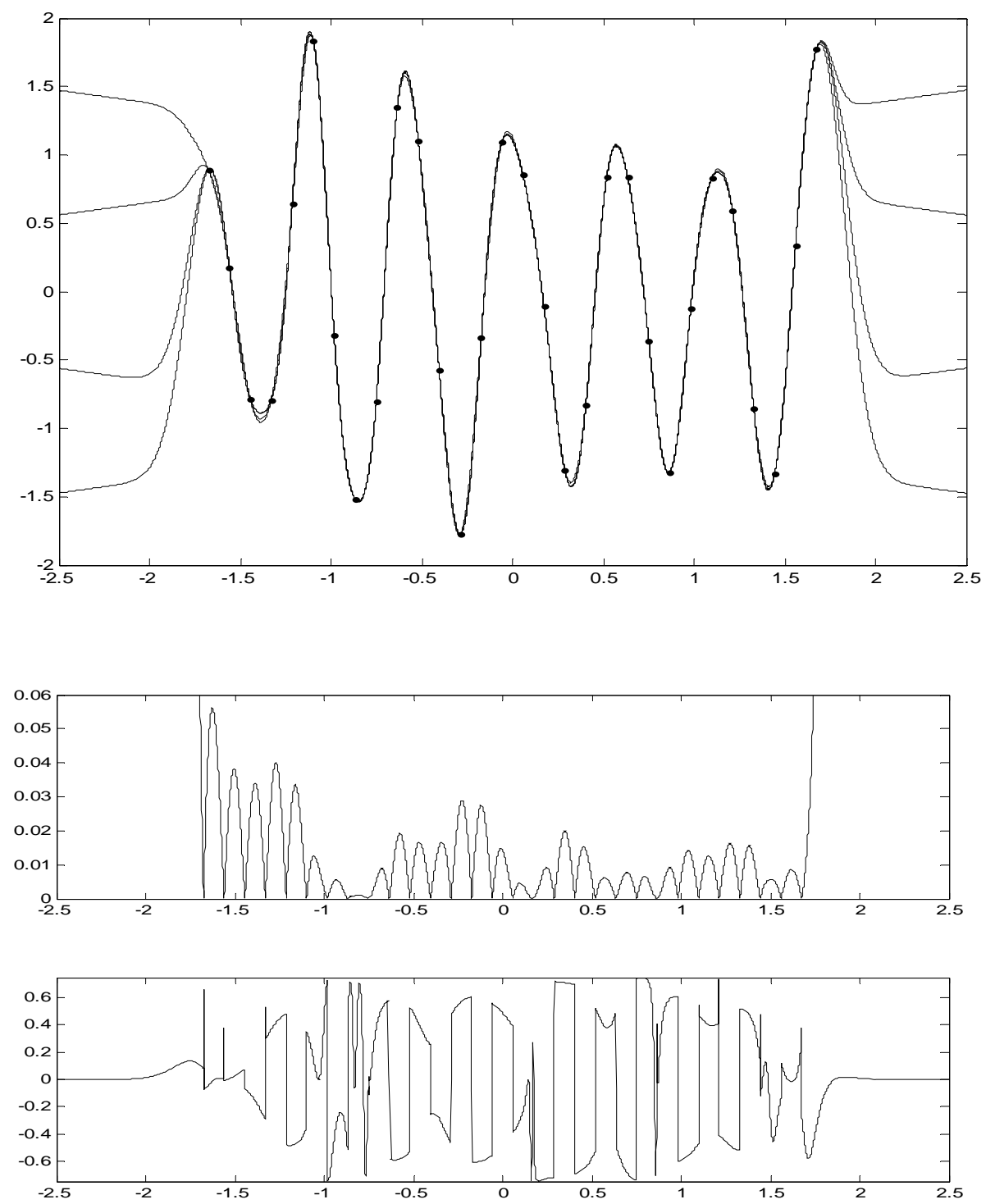

Fig. (4). The top graph shows a communications signal consisting of thirty sample points that are normalized to have zero mean and unit variance in $\mathrm{x}$ and $\mathrm{y}$ and to have the $\mathrm{x}$ axis as their least squares line. As in Fig. (1), the points are interpolated with four (the smallest possible number) of least-rough Gaussian radial basis functions such that their mean and variance extrapolate to those of the classic linear model. The middle and bottom graphs show the standard deviation and the skewness of the basis functions.

\section{REFERENCES}

[1]

C. Like and S. C. Gustafson, "A Framework for Cardinal Modeling", submitted to IEEE Trans. Pattern Anal. Mach. Intell., February 2009.

[2] E. C. Like and S. C. Gustafson, "Robust Cardinal Interpolation", Cybern. Syst. J., Vol. 2, pp. 230-233, July 2008.
[3] S. C. Gustafson, D. R. Parker, and R. K. Martin, "Cardinal Interpolation", IEEE Trans. Pattern Anal. Mach. Intell., vol. 29, pp. 1538-1545, September 2007.

[4] C. Rasmussen and C.K. I. Williams, Gaussian Proc. Mach. Learn., MIT Press, 2006. 